\title{
Astertarone A: A Triterpenoid Ketone Isolated from the Roots of Aster tataricus L.
}

\author{
Toshihiro Akinisa, ${ }^{*, a}$ Yumiko Kimura, ${ }^{b}$ Kazuo Koike, ${ }^{c}$ Takaaki TaI,${ }^{d}$ Ken Yasukawa, ${ }^{b}$ Koichi Arai,${ }^{e}$ \\ Yasuhiro SuzUKI, ${ }^{a}$ and Tamotsu NIKAIDO ${ }^{c}$ \\ College of Science and Technology, Nihon University," 1-8 Kanda Surugadai, Chiyoda-ku, Tokyo 101-8308, Japan, \\ College of Pharmacy, Nihon University, 7-7-1 Narashinodai, Funabashi-shi, Chiba 274-8555, Japan, School of \\ Pharmaceutical Sciences, Toho University, ${ }^{c}$ 2-2-1 Miyama, Funabashi-shi, Chiba 274-8510, Japan, Central Research \\ Laboratory, Kotaro Pharmaceutical Co. Ltd., ${ }^{d}$ 47-3 Suga-cho, Takatsuki-shi, Osaka 569-0022, Japan, and Meikai \\ University School of Dentistry, 1 -1 Keyakidai, Sakado-shi, Saitama 350-0283, Japan. \\ Received July 13, 1998; accepted August 22, 1998
}

The structure of astertarone A isolated from the root extract of Aster tataricus L. (Compositae) was established to be D:A-friedoeuph-21-en-3-one (1) based on spectroscopic methods. This is the first example of a naturally occurring triterpenoid with a D:A-friedoeuphane skeleton. Isolation and identification of epishionol (2), shionone (3), friedelin, epifriedelinol, and $\psi$-taraxasterol are also described.

Key words Aster tataricus; Compositae; roots; triterpenoid ketone; astertarone A

The roots of Aster tataricus L. (Compositae) are used as the Chinese crude drug Asteris Radix (Japanese name: Shion) and are combined in various traditional Chinese prescriptions for use as cough medicines, expectorants and diuretics. ${ }^{1)}$ Constituents of the root extract have been investigated and the presence of a triterpenoid ketone, shionone (shion-21-en-3-one; 3), ${ }^{2-4)}$ seven triterpenoid glycosides, aster saponins $\mathrm{A}-\mathrm{G},{ }^{5-7)}$ and three monoterpenoid glycosides, shionosides $\mathrm{A}-\mathrm{C},{ }^{7,8)}$ have been reported. In this paper, we report the isolation and structure elucidation of a new triterpenoid ketone designated astertarone A (1) from the methanol extract of $A$. tataricus roots. Isolation and characterization of epishionol (shion-21-en-3 $\beta$-ol; 2), a known synthetic triterpene alcohol, ${ }^{2-4,9-11)}$ which has not been isolated before as a natural product, is also described.

Column chromatography of the hexane soluble portion of the methanol extract of $A$. tataricus roots on silica gel afforded the triterpenoid ketone and triterpene alcohol fractions. Crystallization of the ketone fraction from acetonemethanol followed by preparative HPLC of the filtrate portion eventually yielded compound $\mathbf{1}$ in addition to $3^{2-4)}$ and friedelin (D: A-friedoolean-3-one). ${ }^{12)}$

Compound 1, molecular formula $\mathrm{C}_{30} \mathrm{H}_{500}$ determined from its high-resolution mass spectrum (HR-MS) $\left([\mathrm{M}]^{+}, \mathrm{m} / z\right.$ 426.3848), gave IR absorptions at 1712 (ketone) and 824 $\mathrm{cm}^{-1}$ (trisubstituted double bond). Compound 1 displayed four methyl singlets at $\delta 0.72,0.79,0.86$, and 0.87 , two methyl doublets at $\delta 0.86(J=6.5 \mathrm{~Hz})$ and $0.88(J=6.6 \mathrm{~Hz})$, two olefinic methyl singlets at $\delta 1.61$ and 1.69 , with an olefinic methine signal at $\delta 5.09(\mathrm{tt}, J=1.4,6.9 \mathrm{~Hz})$ in the ${ }^{1} \mathrm{H}-\mathrm{NMR}$ spectrum. These data, in combination with MS fragment ions having $\mathrm{m} / \mathrm{z} 313$ (base peak) [loss of side-chain $\left(\mathrm{C}_{8} \mathrm{H}_{15}\right)$ with $2 \mathrm{H}$ transfer], 273 [loss of side-chain and ring $\mathrm{D}$ $\left.\left(\mathrm{C}_{3} \mathrm{H}_{6}\right)\right]$, and $69\left[\mathrm{CH}_{2} \mathrm{CH}=\mathrm{C}(\mathrm{Me})_{2}\right]^{+}(\mathrm{C}-20-\mathrm{C}-22$, and $\mathrm{C}-29$ and $\mathrm{C}-30),{ }^{13)}$ suggested that compound 1 has a $\mathrm{C}_{8}$ side-chain containing an isopropylidene group, like euphol (eupha-8,24dien- $3 \beta$-ol) and tirucallol (tirucalla- $8,24-$ dien- $3 \beta$-ol), ${ }^{14)}$ and a tetracyclic triterpenoid skeleton with a keto group most probably located at C-3. Moreover, the presence as a secondary methyl group of one of the five skeletal methyl groups sug-

* To whom correspondence should be addressed. gested it to possess a D:A-friedoeuphane or tirucallane skeleton. From the foregoing, compound 1 was assigned a D:Afriedoeuph/tirucall-21-en-3-one structure with as-yet-to-be determined stereochemistry. Analysis of the ${ }^{13} \mathrm{C}$ distortionless enhancement by polarization transfer (DEPT), ${ }^{1} \mathrm{H}-{ }^{1} \mathrm{H}$ correlation spectroscopy (COSY), ${ }^{1} \mathrm{H}$ detected multiple quantum coherence (HMQC), and heteronuclear multiplebond correlation (HMBC) spectra, and the ${ }^{13} \mathrm{C}$ - and ${ }^{1} \mathrm{H}$ NMR spectral comparison of $\mathbf{1}$ (Table 1) with $\mathbf{3}$ (see the Experimental section), friedelin, ${ }^{12)}$ and euphol and tirucallol acetates $^{14)}$ confirmed the above assumption.

The stereochemistry of compound 1 was established by phase-sensitive nuclear Overhauser and exchange spectroscopy (NOESY). Compound 1 showed significant NOE correlations between $[\mathrm{H}-4 \alpha-\mathrm{H}-10 \alpha]$ and $[\mathrm{H}-23(4 \beta-\mathrm{Me})-\mathrm{H}-$ $24(5 \beta-\mathrm{Me})-\mathrm{H}-25$ (9 $\beta$-Me)-H-7 $\beta$-H-26 (14 $\beta$-Me)] (Fig. 1), which were observed also for compound 3 (see the Experimental section), demonstrating that 1 possessed the same stereochemistry as $\mathbf{3}$ as far as rings $\mathrm{A}, \mathrm{B}$ and $\mathrm{C}$ and the $\mathrm{C} / \mathrm{D}$ ring junction $(13 \alpha, 14 \beta)$ were concerned. In addition, compound 1 exhibited NOE correlations between [H-26 (14 $\beta$ Me)-H-17-H-28], [H-28-H-16 $\alpha, \beta]$, and [H-16 $\alpha-\mathrm{H}-18-\mathrm{H}-$ $27(13 \alpha-\mathrm{Me})]$ which were consistent with those observed for euphol acetate. ${ }^{13)}$ From these data, it was concluded that 1 was D:A-friedoeuph-21-en-3-one with a $18 R$-chirality which

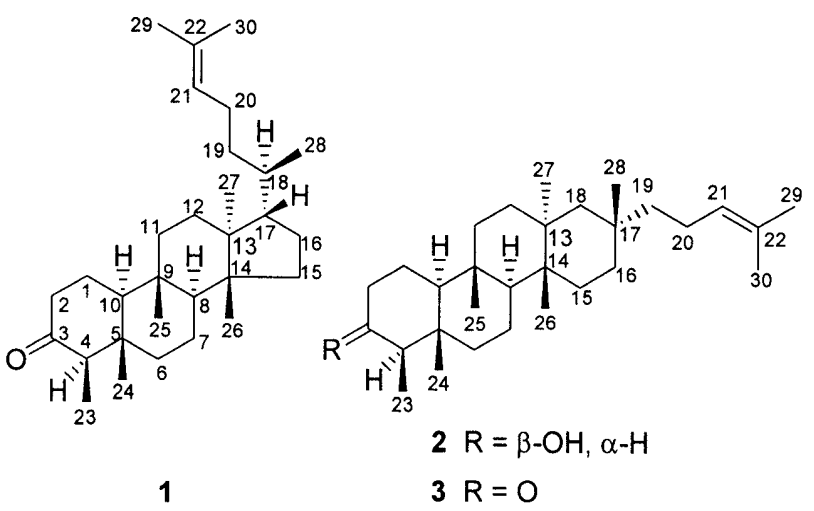

Chart 1. Structures of Triterpenoids from Aster tataricus Roots

(C) 1998 Pharmaceutical Society of Japan 
Table $1 .{ }^{13} \mathrm{C}-\mathrm{NMR}$ and ${ }^{1} \mathrm{H}$-NMR Spectral Data $\left(\delta\right.$ Values; $\left.\mathrm{CDCl}_{3}\right)$ and ${ }^{1} \mathrm{H}-{ }^{13} \mathrm{C}$ Long-Range Correlations of Astertarone $\mathrm{A}(\mathbf{1})$ by ${ }^{1} \mathrm{H}-{ }^{1} \mathrm{H}$ COSY, $\mathrm{HMBC}$ and HMQC

\begin{tabular}{|c|c|c|c|c|}
\hline \multirow{2}{*}{$\frac{\text { C No. }}{1}$} & \multicolumn{2}{|c|}{$\delta_{\mathrm{C}}$} & \multirow{2}{*}{$\frac{\delta_{\mathrm{H}}{ }^{a)}}{1.96(\alpha), 1.74(\beta)}$} & \multirow[t]{2}{*}{ Cross peaks $\left(\delta_{\mathrm{C}}\right)$ in $\mathrm{HMBC}$ spectrum } \\
\hline & 22.8 & $\mathrm{CH}_{2}$ & & \\
\hline 2 & 41.6 & $\mathrm{CH}_{2}$ & $2.30(\alpha), 2.39(\beta)$ & \\
\hline 3 & 213.2 & $\mathrm{C}^{2}$ & & \\
\hline 4 & 58.3 & $\mathrm{CH}$ & 2.28 & $6.8(\mathrm{C}-23), 14.7(\mathrm{C}-24), 42.4(\mathrm{C}-5), 213.2(\mathrm{C}-3)$ \\
\hline 5 & 42.4 & $\mathrm{C}$ & & \\
\hline 6 & 40.8 & $\mathrm{CH}_{2}$ & $1.32(\alpha), 1.72(\beta)$ & \\
\hline 7 & 20.3 & $\mathrm{CH}_{2}$ & $1.29(\alpha), 1.57(\beta)$ & \\
\hline 8 & 50.0 & $\mathrm{CH}^{2}$ & 1.58 & $16.0(\mathrm{C}-26), 18.5(\mathrm{C}-25), 20.3(\mathrm{C}-7), 37.9(\mathrm{C}-9), 59.1$ (C-10) \\
\hline 9 & 37.9 & $\mathrm{C}$ & & \\
\hline 10 & 59.1 & $\mathrm{CH}$ & 1.60 & $18.5(\mathrm{C}-25), 22.8(\mathrm{C}-1), 36.8(\mathrm{C}-11), 41.6(\mathrm{C}-2), 42.4(\mathrm{C}-5)$ \\
\hline 11 & 36.8 & $\mathrm{CH}_{2}$ & $1.43(2 \mathrm{H})$ & \\
\hline 12 & 34.0 & $\mathrm{CH}_{2}^{2}$ & $1.22(\alpha), 1.14(\beta)$ & \\
\hline 13 & 46.2 & $\mathrm{C}^{2}$ & & \\
\hline 14 & 48.2 & $\mathrm{C}$ & & \\
\hline 15 & 30.1 & $\mathrm{CH}_{2}$ & $1.76(\alpha), 1.56(\beta)$ & \\
\hline 16 & 28.1 & $\mathrm{CH}_{2}$ & $1.30(\alpha), 1.89(\beta)$ & \\
\hline 17 & 49.8 & $\mathrm{CH}^{2}$ & 1.46 & $19.3(\mathrm{C}-27), 35.3(\mathrm{C}-18), 46.2(\mathrm{C}-13)$ \\
\hline 18 & 35.3 & $\mathrm{CH}$ & 1.52 & $19.0(\mathrm{C}-28), 35.4(\mathrm{C}-19)$ \\
\hline 19 & 35.4 & $\mathrm{CH}_{2}$ & $1.11,1.61$ & \\
\hline 20 & 24.7 & $\mathrm{CH}_{2}$ & $1.87,2.04$ & \\
\hline 21 & 125.2 & $\mathrm{CH}^{2}$ & $5.09(\mathrm{tt}, 1.4,6.9)$ & \\
\hline 22 & 131.0 & $\mathrm{C}$ & & \\
\hline 23 & 6.8 & $\mathrm{Me}$ & $0.88(\mathrm{~d}, 6.6)$ & $14.7(\mathrm{C}-24), 58.3(\mathrm{C}-4), 213.2(\mathrm{C}-3)$ \\
\hline 24 & 14.7 & $\mathrm{Me}$ & $0.72(\mathrm{~s})$ & $40.8(\mathrm{C}-6), 42.4(\mathrm{C}-5), 58.3(\mathrm{C}-4), 59.1(\mathrm{C}-10)$ \\
\hline 25 & 18.5 & $\mathrm{Me}$ & $0.86(\mathrm{~s})$ & $36.8(\mathrm{C}-11), 37.9(\mathrm{C}-9), 50.0(\mathrm{C}-8), 59.1(\mathrm{C}-10)$ \\
\hline 26 & 16.0 & $\mathrm{Me}$ & $0.87(\mathrm{~s})$ & $30.1(\mathrm{C}-15), 42.4(\mathrm{C}-5), 46.2(\mathrm{C}-13), 48.2(\mathrm{C}-14)$ \\
\hline 27 & 19.3 & $\mathrm{Me}$ & $0.79(\mathrm{~s})$ & $34.0(\mathrm{C}-12), 46.2(\mathrm{C}-13), 48.2(\mathrm{C}-14), 49.8(\mathrm{C}-17)$ \\
\hline 28 & 19.0 & $\mathrm{Me}$ & $0.86(\mathrm{~d}, 6.5)$ & 35.3 (C-18), 35.4 (C-19), 49.8 (C-17), 50.0 (C-8) \\
\hline 29 & 25.8 & $\mathrm{Me}$ & $1.69(\mathrm{~s})$ & $17.7(\mathrm{C}-30), 125.2(\mathrm{C}-21), 131.0(\mathrm{C}-22)$ \\
\hline 30 & 17.7 & $\mathrm{Me}$ & $1.61(\mathrm{~s})$ & $25.8(\mathrm{C}-29), 125.2(\mathrm{C}-21), 131.0(\mathrm{C}-22)$ \\
\hline
\end{tabular}

a) Figures in parentheses in the ${ }^{1} \mathrm{H}$ chemical shift column denote $J$ values $(\mathrm{Hz})$.

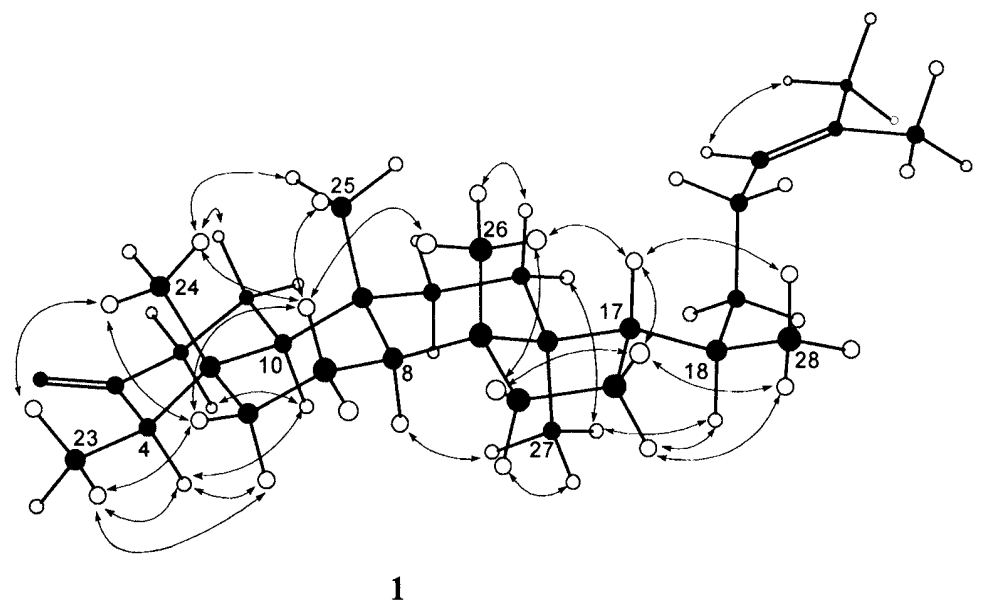

Fig. 1. Energy-Minimized Conformations and Some Representative NOE Correlations $(\leftrightarrow)$ for Astertarone A (1)

we named astertarone A.

The most stable conformation of $\mathbf{1}$ was simulated using MacroModel. The results of the calculations ${ }^{15,16)}$ are shown in Fig. 1 together with the significant NOE's $(\leftrightarrow)$. The conformer of simulated 1 shows C-19 of the side chain at C-17 oriented into a "left-handed" conformation (C-19 trans-oriented to C-13) similar to that of butyrospermol (eupha-7,24dien-3 $\beta$-ol) acetate ${ }^{17)}$ and the crystal structure of euphol acetate. ${ }^{18)}$ This was consistent with the NOE experiment done in solution, thus confirming the proposed structure of 1.

Preparative HPLC of the triterpene alcohol fraction yielded epishionol (2), ${ }^{2-4,9-11)}$ along with epifriedelinol
(D:A-friedoolean-3 $\beta$-ol $)^{2,10,11)}$ and $\psi$-taraxasterol (taraxast20 -en-3 $\beta$-ol). ${ }^{19)}$

Astertarone A (1) is the first naturally occurring triterpenoid possessing a D:A-friedoeuphane skeleton. A triterpenoid hydrocarbon possessing a closely related structure with 1, named aonena-3,21-diene with a $(17 \xi, 18 \mathrm{~S})-\mathrm{D}: \mathrm{A}-$ friedotirucallane-type skeleton, has been isolated from the rhizomes of Polypodiodes niponica. ${ }^{20)}$ Although epishionol (2) has previously been synthesized from shionone (3) by reduction, ${ }^{2-4,9-11)}$ this is the first report for its detection as a natural product. 


\section{Experimental}

Crystallizations were performed from acetone-MeOH. Melting points measured were uncorrected. Reverse-phase HPLC was carried out on an octadecyl silica column (Superiorex ODS S- $5 \mu \mathrm{m}$ column, $10 \mathrm{~mm}$ i.d. $\times 25 \mathrm{~cm}$; Shiseido Co., Ltd., Tokyo) at $25^{\circ} \mathrm{C}$ with $\mathrm{MeOH}(4 \mathrm{ml} / \mathrm{min})$ as mobile phase. GLC was performed using a DB-17 fused-silica capillary column $\left(30 \mathrm{~m} \times 0.3 \mathrm{~mm}\right.$ i.d., column temp., $\left.275^{\circ} \mathrm{C}\right)$. In both HPLC and GLC, cholesterol (cholest-5-en-3 $\beta$-ol) was the standard for the determination of $\mathrm{R} t_{\mathrm{R}}$ of triterpenoids. Electron-impact MS and HR-MS were recorded at $70 \mathrm{eV}$. NMR spectra were recorded at $400 \mathrm{MHz}\left({ }^{1} \mathrm{H}-\mathrm{NMR}\right)$ and $100.6 \mathrm{MHz}\left({ }^{13} \mathrm{C}\right.$ $\mathrm{NMR}$ ) in $\mathrm{CDCl}_{3}$ with tetramethylsilane (TMS) ( ${ }^{1} \mathrm{H}-\mathrm{NMR}$ ) and $\mathrm{CDCl}_{3}$ at $\delta$ $77.0\left({ }^{13} \mathrm{C}\right.$-NMR) as internal standard, and chemical shifts were recorded in $\delta$ values. IR spectra were recorded in $\mathrm{KBr}$. Instrumental details were the same as described previously. ${ }^{21)}$ The dried roots of $A$. tataricus were purchased from Tochimoto Co., Ltd. (Osaka). Identification of the plant material was performed by Prof. Norio Sahashi (Department of Biology, School of Pharmaceutical Sciences, Toho University). Friedelin ${ }^{12)}$ and $\psi$-taraxasterol ${ }^{19)}$ were used as reference compounds.

Isolation Procedures Dried and ground $A$. tataricus roots $(2.5 \mathrm{~kg})$ were extracted $3 \times$ for $3 \mathrm{~d}$ with $\mathrm{MeOH}$ (6 1 each) at room temperature to give an extract $(600 \mathrm{~g})$ which was partitioned between EtOAc- $\mathrm{H}_{2} \mathrm{O}$ to afford an EtOAc fraction $(34.5 \mathrm{~g})$. This extract was partitioned between hexane$\mathrm{MeOH}-\mathrm{H}_{2} \mathrm{O}(19: 19: 2)$. The hexane fraction $(19.9 \mathrm{~g})$ was subjected to column chromatography $(\mathrm{CC})$ on silica gel $(600 \mathrm{~g})$ using hexane and a hexane-EtOAc gradient of $50: 1-1: 1$, which yielded triterpenoid ketone (A) and triterpene alcohol (B) fractions. Repeated CC of the fractions using the same solvent system eventually yielded purified fractions $\mathrm{A}(1.8 \mathrm{~g})$ and $\mathrm{B}$ $(0.5 \mathrm{~g})$. Recrystallization of the fraction A from acetone- $\mathrm{MeOH}$ gave crystallized (1.2 g), constituted with shionone (3), and filtrate portions $(360 \mathrm{mg})$. Preparative HPLC of the filtrate portion yielded astertarone A $[1 ; 5 \mathrm{mg}$; amorphous solid; $\mathrm{R} t_{\mathrm{R}}: 1.09$ (HPLC), 2.28 (GLC)], 3 [227 mg; mp 162$164^{\circ} \mathrm{C}$ (colorless needles) (lit., ${ }^{2)} \mathrm{mp} 158.5-159.5^{\circ} \mathrm{C}$; lit., ${ }^{8}{ }^{8} \mathrm{mp} 155-$ $\left.156^{\circ} \mathrm{C}\right) ; \mathrm{R} t_{\mathrm{R}}: 1.19$ (HPLC), 2.54 (GLC)], and friedelin [32 mg; mp 266$269^{\circ} \mathrm{C}$ (colorless plates) (lit., ${ }^{12)} \mathrm{mp} 268-269^{\circ} \mathrm{C}$ ); $\mathrm{R} t_{\mathrm{R}}: 1.48$ (HPLC), 3.05 (GLC)]. On the other hand, preparative HPLC of the fraction B afforded epishionol [2; $10 \mathrm{mg}$; mp $116-119^{\circ} \mathrm{C}$ (colorless needles) (lit., ${ }^{2)} \mathrm{mp} 117.5-$ $118.5^{\circ} \mathrm{C}$ ); $\mathrm{R}_{\mathrm{R}}: 1.15$ (HPLC), 2.32 (GLC)], epifriedelinol [ $35 \mathrm{mg} ; \mathrm{mp} 275$ $279^{\circ} \mathrm{C}$ (colorless plates) (lit., ${ }^{2)} \mathrm{mp} 279-283^{\circ} \mathrm{C}$; lit., ${ }^{8)} \mathrm{mp} 299^{\circ} \mathrm{C}$ ); $\mathrm{R} t_{\mathrm{R}}: 1.54$ (HPLC), 2.76 (GLC)], and $\psi$-taraxasterol [67 mg; mp 290-292 ${ }^{\circ} \mathrm{C}$ (colorless plates); $\mathrm{R} t_{\mathrm{R}}: 1.23$ (HPLC), 2.72 (GLC)]. Identification of $\left.\mathbf{2},{ }^{10,11}\right) \mathbf{3}^{10,11)}$ and epifriedelinol ${ }^{10,11)}$ was based on spectroscopic (MS and ${ }^{1} \mathrm{H}-\mathrm{NMR}$ ) comparison with data in the literature, whereas that of friedelin and $\psi$-taraxasterol was done by chromatographic (HPLC, GLC) and spectroscopic (MS, ${ }^{1} \mathrm{H}-\mathrm{NMR}$ ) comparison with reference compounds. Since the fully assigned ${ }^{1} \mathrm{H}$ - and ${ }^{13} \mathrm{C}$-NMR spectral data for $\mathbf{2}$ and $\mathbf{3}$ were unavailable in the literature, these also are shown below accompanied by some representative NOE correlations. The NMR assignments were aided by ${ }^{13} \mathrm{C}$ DEPT, ${ }^{1} \mathrm{H}-{ }^{1} \mathrm{H}$ COSY, HMQC, HMBC, and phase-sensitive NOESY spectroscopy.

Astertarone A (1): IR $v_{\max } \mathrm{cm}^{-1}: 1712,824$. MS $\mathrm{m} / z(\%): 426\left(\mathrm{M}^{+}, 13\right)$ 411 (21), 341 (19), 313 (100), 273 (3), 245 (3), 218 (14), 203 (7), 191 (10), 189 (6), 69 (97). HR-MS: $m / z$ 426.3848 [Calcd for $\mathrm{C}_{30} \mathrm{H}_{50} \mathrm{O}\left(\mathrm{M}^{+}\right)$: 426.3859]; 313.2506 [Calcd for $\mathrm{C}_{22} \mathrm{H}_{33} \mathrm{O}: 313.2528$ ]; 273.2243 [Calcd for $\mathrm{C}_{19} \mathrm{H}_{29} \mathrm{O}: 273.2217$ ]; 69.0697 [Calcd for $\mathrm{C}_{5} \mathrm{H}_{9}:$ 69.0703]. See Table 1 for the ${ }^{13} \mathrm{C}$ - and ${ }^{~} \mathrm{H}-\mathrm{NMR}$ data.

Epishionol (2): ${ }^{13} \mathrm{C}$ - and ${ }^{1} \mathrm{H}-\mathrm{NMR}: \mathrm{C}-1\left[\delta_{\mathrm{C}} 15.8 ; \delta_{\mathrm{H}} 1.46(\alpha), 1.56(\beta)\right]$ C-2 [35.2; $1.57(\alpha), 1.90(\beta)], \mathrm{C}-3[72.8 ; 3.73, \mathrm{dt}, J=2.5,3.1 \mathrm{~Hz}], \mathrm{C}-4[49.2$; $1.25], \mathrm{C}-5[37.9], \mathrm{C}-6[41.7 ; 0.95(\alpha), 1.72(\beta)], \mathrm{C}-7[17.3 ; 1.26(\alpha), 1.37$ $(\beta)$ ], C-8 [50.0; 1.22], C-9 [38.2], C-10 [61.1;0.96], C-11 [35.3; $1.39(2 \mathrm{H})]$, C-12 [32.5; $0.89(\alpha), 1.53(\beta)], \mathrm{C}-13$ [36.9], C-14 [38.7], C-15 [29.2; 1.30 $(\alpha), 1.24(\beta)], \mathrm{C}-16[34.8 ; 1.33(\alpha), 1.60(\beta)], \mathrm{C}-17[31.8], \mathrm{C}-18[44.7 ; 1.13$ $(\alpha), 1.19(\beta)], \mathrm{C}-19$ [43.6; 1.16, 1.67], C-20 [23.6; 1.85, 1.99], C-21 [125.4; $5.10, \mathrm{tt}, J=1.5,7.3 \mathrm{~Hz}], \mathrm{C}-22$ [130.7], C-23 [11.6; 0.93, d, $J=7.0 \mathrm{~Hz}], \mathrm{C}-24$ $[16.4 ; 0.95, \mathrm{~s}], \mathrm{C}-25[20.0 ; 0.92, \mathrm{~s}], \mathrm{C}-26[15.1 ; 0.87, \mathrm{~s}], \mathrm{C}-27[20.7 ; 1.09$, s], C-28 [33.0; 0.89, s], C-29 [25.7; 1.68, s], C-30 [17.6; 1.60, s]. Significant NOE correlations observed were between $[\mathrm{H}-3 \alpha-\mathrm{H}-4 \alpha-\mathrm{H}-10 \alpha-\mathrm{H}-8 \alpha-\mathrm{H}-$ $27(13 \alpha-\mathrm{Me})],[\mathrm{H}-23(4 \beta-\mathrm{Me})-\mathrm{H}-6 \beta-\mathrm{H}-24(5 \beta-\mathrm{Me})]$, [H-6 $\beta-\mathrm{H}-7 \beta-\mathrm{H}-25$
(9 $\beta$-Me)], and $[\mathrm{H}-7 \beta-\mathrm{H}-26(14 \beta-\mathrm{Me})-\mathrm{H}-18 \beta-\mathrm{H}-28(17 \beta-\mathrm{Me})]$.

Shionone $(3):{ }^{13} \mathrm{C}$ - and ${ }^{1} \mathrm{H}-\mathrm{NMR}$ : $\mathrm{C}-1\left[\delta_{\mathrm{C}} 22.3 ; \delta_{\mathrm{H}} 1.97(\alpha), 1.70(\beta)\right]$, C$2[41.5 ; 2.40(\alpha), 2.30(\beta)], \mathrm{C}-3[213.1], \mathrm{C}-4[58.2 ; 2.25, \mathrm{q}, J=6.2 \mathrm{~Hz}], \mathrm{C}-5$ [42.2], C-6 [41.2; $1.24(\alpha), 1.73(\beta)]$, C-7 [17.9; $1.47(\alpha), 1.35(\beta)]$, C-8 $[50.0 ; 1.32], \mathrm{C}-9$ [38.5], C-10 [59.7; 1.58$], \mathrm{C}-11[35.3 ; 1.40,1.52], \mathrm{C}-12$ [32.3; $0.92(\alpha), 1.58(\beta)], \mathrm{C}-13$ [36.9], C-14 [38.6], C-15 [29.3; $1.34(\alpha)$, $1.27(\beta)], \mathrm{C}-16[34.7 ; 1.36(\alpha), 1.59(\beta)], \mathrm{C}-17$ [31.7], C-18 [44.6; $1.12(\alpha)$ $1.22(\beta)], \mathrm{C}-19[43.6 ; 1.17,1.67], \mathrm{C}-20[23.2 ; 1.83,2.00], \mathrm{C}-21[125.3$; $5.10, \mathrm{ttt}, J=1.3,1.5,7.2 \mathrm{~Hz}], \mathrm{C}-22[130.8], \mathrm{C}-23[6.8 ; 0.88, \mathrm{~d}, J=6.6 \mathrm{~Hz}], \mathrm{C}-$ 24 [14.6; 0.72, s], C-25 [19.6; 0.93, s], C-26 [15.2; 0.90, s], C-27 [20.6; $1.14, \mathrm{~s}], \mathrm{C}-28[33.0 ; 0.91, \mathrm{~s}], \mathrm{C}-29[25.7 ; 1.69, \mathrm{~s}], \mathrm{C}-30[17.6 ; 1.61, \mathrm{~s}]$. The following diagnostic NOE correlations were observed between $[\mathrm{H}-4 \alpha-\mathrm{H}-$ $10 \alpha-\mathrm{H}-8 \alpha, \mathrm{H}-11 \alpha-\mathrm{H}-27(13 \alpha-\mathrm{Me})]$ and $[\mathrm{H}-23(4 \beta-\mathrm{Me})-\mathrm{H}-24(5 \beta-\mathrm{Me})-\mathrm{H}-$ $25(9 \beta-\mathrm{Me})-\mathrm{H}-7 \beta-\mathrm{H}-26(14 \beta-\mathrm{Me})-\mathrm{H}-18 \beta-\mathrm{H}-28(17 \beta-\mathrm{Me})]$

Acknowledgements The authors are indebted to Prof. Norio Sahashi (Toho University) for the identification of the plant material.

\section{References and Notes}

1) Namba T., "The Encyclopedia of Wakan-Yaku (Traditional SinoJapanese Medicines) with Color Pictures," Revised edn., Vol. I, Hoikusha, Osaka, 1994, p. 161.

2) Takahashi M., Kamisako W., Ishimasa S., Miyamura K., Yakugaku Zasshi, 79, 1281-1283 (1959).

3) Tanahashi Y., Takahashi T., Patil F., Ourisson G., Bull. Soc. Chim. Fr., 1964, 584-590.

4) Tanahashi Y., Moriyama Y., Takahashi T., Bull. Soc. Chim. Fr., 1966, $1670-1677$

5) Nagao T., Hachiyama S., Okabe H., Yamauchi T., Chem. Pharm. Bull., 37, 1977-1983 (1989).

6) Nagao T., Okabe H., Yamauchi T., Chem. Pharm. Bull., 38, 783-785 (1990)

7) Cheng D., Shao Y., Phytochemistry, 35, 173-176 (1994).

8) Nagao T., Okabe H., Yamauchi T., Chem. Pharm. Bull., 36, 571-577 (1988)

9) Tanahashi Y., Moriyama Y., Takahashi T., Patil F., Ourisson G., Bull. Soc. Chim. Fr., 1966, 2374-2377.

10) Hirota H., Moriyama Y., Tsuyuki T., Tanahashi Y., Takahashi T., Katoh Y., Satoh H., Bull. Chem. Soc. Jpn., 48, 1884-1888 (1975)

11) Kikuchi T., Yokoi T., Niwa M., Shingu T., Chem. Pharm. Bull., 28, 2014-2023 (1980)

12) Akihisa T., Yamamoto K., Tamura T., Kimura Y., Iida T., Nambara T., Chang F. C., Chem. Pharm. Bull., 40, 789-791 (1992).

13) Goad L. J., Akihisa T., "Analysis of Sterols," Blackie Academic and Professional, London, 1997.

14) Akihisa T., Kimura Y., Koike K., Shibata T., Yoshida Z., Nikaido T., Tamura T., J. Nat. Prod., 61, 409-412 (1998).

15) Calculations were performed using MacroModel Ver. 6.0 with extended MM3 parameters. Conformation with minimum steric energy was obtained through a Metropolis Monte Carlo procedure. Final structures were depicted using Chem3D program (Cambridge Scientific Computing Inc., Cambridge, MA).

16) Mohamadi F., Richards N. G. L., Guida W. C., Liskamp R., Lipton M., Caufield C., Chang G., Hendrickson T., Still W. C., J. Comput. Chem., 11, 440-467 (1990)

17) Akihisa T., Kimura Y., Kokke W. C. M. C., Takase S., Yasukawa K., Tamura T., J. Chem. Soc., Perkin Trans. 1, 1996, 2379-2384.

18) Nes W. D., Wong R. Y., Benson J. R., Landrey J. R., Nes W. R., Proc. Natl. Acad. Sci. U.S.A., 81, 5896-5900 (1984).

19) Akihisa T., Yasukawa K., Oinuma H., Kasahara Y., Yamanouchi S., Takido M., Kumaki K., Tamura T., Phytochemistry, 43, 1255-1260 (1996)

20) Arai Y., Hirohata M., Ageta H., Tetrahedron Lett., 30, 7209-7212 (1989).

21) Akihisa T., Kimura Y., Kokke W. C. M. C., Itoh T., Tamura T., Chem. Pharm. Bull., 44, 1202-1207 (1996). 